\title{
CONTRIBUCIÓN AL CONOCIMIENTO DE LA MICOBIOTA DE LA REGIÓN DE SAN SEBASTIÁN DEL OESTE, JALISCO, MÉXICO
}

\author{
María de Jesús Herrera Fonseca \\ Laura GuZmán-Dávalos \\ Y \\ Olivia RodríGuez \\ Instituto de Botánica \\ Departamento de Botánica y Zoología \\ Universidad de Guadalajara \\ Apartado postal 1-139 \\ 45101 Zapopan, Jalisco
}

\begin{abstract}
RESUMEN
Se presenta un inventario preliminar de los hongos, la mayor parte de ellos macroscópicos, del municipio de San Sebastián del Oeste, Jalisco, estudiados a través de tres años. El área está cubierta por bosques de pino, encino, mesófilo de montaña, tropical caducifolio y subcaducifolio, con algunas zonas agrícolas. De los 583 ejemplares estudiados, se determinaron 216 especies, de ellas $89.4 \%$ corresponde a Basidiomycota, $8.8 \%$ a Ascomycota, $1.3 \%$ a Myxomycota, y $0.5 \%$ a Deuteromycota. 35 registros son nuevos para Jalisco y 2 especies se citan por primera vez para México: Amanita roseitincta (Murrill) Murrill y Ramaria abietina (Pers. : Fr.).

Palabras clave: hongos, San Sebastián del Oeste, Jalisco, México.

ABSTRACT

A preliminary inventory of fungi, most of them macrofungi, from the municipality of San Sebastián del Oeste, Jalisco, Mexico, studied in three years, is presented. The area is largely characterized by pine, oak, mesophytic, tropical deciduous and tropical subdeciduous forests with few agriculture zones. Based on 583 specimens, 216 species were identified, of which $89.4 \%$ correspond to Basidiomycota, $8.8 \%$ to Ascomycota, $1.3 \%$ to Myxomycota and $0.5 \%$ to Deuteromycota. Furthermore 35 new records for the state of Jalisco and two new species for Mexico: Amanita roseitincta (Murrill) Murrill and Ramaria abietina (Pers. : Fr.), are documented.
\end{abstract}

Key words: fungi, San Sebastián del Oeste, Jalisco, Mexico.

\section{INTRODUCCIÓN}

A pesar del esfuerzo en los últimos años por incrementar el conocimiento de la micobiota de México, aún falta mucho por conocer en varias regiones del país. En Jalisco los estudios sobre el grupo se iniciaron por Guzmán y García-Saucedo (1973) y Manzi 
(1976). Hasta ahora son muy pocos los trabajos que han realizado inventarios en algunas regiones del estado. Nieves (1985) elaboró un listado de los macromicetos del bosque La Primavera, Téllez et al. (1988) describieron los hongos de la reserva de la biosfera de la Sierra de Manantlán y Vázquez y Guzmán-Dávalos (1988) registraron los hongos macroscópicos de la Barranca de Huentitán. Rodríguez et al. (1994) hicieron un censo preliminar de los hongos del Volcán de Tequila y Fierros y Guzmán-Dávalos (1995) efectuaron un inventario de los macromicetos de la Sierra de Quila. Además se han llevado a cabo trabajos taxonómicos de géneros, como Scleroderma (Guzmán-Dávalos y Guzmán, 1985), Volvariella (Vázquez et al., 1989; Vázquez y Guzmán-Dávalos, 1991c), Gymnopilus (Guzmán-Dávalos y Guzmán, 1986; Guzmán-Dávalos y Guzmán, 1991), Pluteus (Rodríguez y Guzmán-Dávalos, 1997; Rodríguez et al.,1997; Rodríguez, 1998), Thelephora (SánchezJácome y Guzmán-Dávalos, 1997) y Psilocybe (Guzmán, 1998).

En cuanto al estudio de la micobiota de la región de San Sebastián del Oeste, se tienen tan sólo 9 citas aisladas. Guzmán-Dávalos y Guzmán (1985) registraron a Scleroderma texense, recolectada en bosque de pino-encino; Vázquez y Guzmán-Dávalos (1991a) citaron a Porodisculus pendulus; Rodríguez y Guzmán-Dávalos (1993) mencionaron a Megasporoporia mexicana, especie más o menos común en bosque de pino-encino; por su parte Sánchez-Jácome y Guzmán-Dávalos (1997) registraron a Thelephora arbuscula y $T$. vialis de bosque de encino-pino, con influencia tropical. Recientemente Rodríguez (1998) citó a Pluteus petasatus, de bosque tropical subcaducifolio y por último Guzmán (1998) a Psilocybe coprophila de dos localidades del municipio de San Sebastián del Oeste y describió como nuevas especies para la ciencia a $P$. villarrealiae y $P$. laurae, recolectadas a la orilla del camino en bosque mesófilo de montaña.

El municipio de San Sebastián del Oeste representa 1.55\% de la superficie del estado de Jalisco con una extensión de 23,604 has (Anónimo, 1988) (Fig. 1). Debido a su localización estratégica en el extremo noroccidental del Eje Neovolcánico y a su carácter montañoso, se encuentran microhábitats únicos y variados (Reynoso y Curiel, 1997). Ésta es una condición favorable para la riqueza biológica de la zona, que se debe en parte al buen estado de conservación de la vegetación, en función de su aislamiento relativo, debido principalmente a la falta de carreteras. Por tal razón actualmente se están haciendo esfuerzos para que la región de San Sebastián del Oeste, junto con la costa norte de Jalisco, sea decretada como área protegida.

\section{MATERIALES Y MÉTODOS}

El material estudiado fue recolectado durante 1995 con motivo de la XIV Exposición de Hongos de Jalisco, llevada a cabo en la población de San Sebastián del Oeste del 15 al 17 de septiembre. Muestreos adicionales en 1997 y 1998 se efectuaron de junio a octubre. Los tipos de vegetación explorados fueron los bosques de pino, encino, pino-encino y mesófilo de montaña, con altitudes que oscilan de $1300-2290 \mathrm{~m}$, y los bosques tropical caducifolio y subcaducifolio, con altitudes de 550-760 m. El material se encuentra depositado en el herbario IBUG. En total se obtuvieron 1060 ejemplares.

Para el estudio microscópico del material revisado se siguieron las técnicas tradicionales utilizadas en micología (Largent et al., 1977), realizándose cortes del cuerpo fructífero y preparaciones montadas en $\mathrm{KOH}$ a $5 \%$. En algunos casos se usó el reactivo 


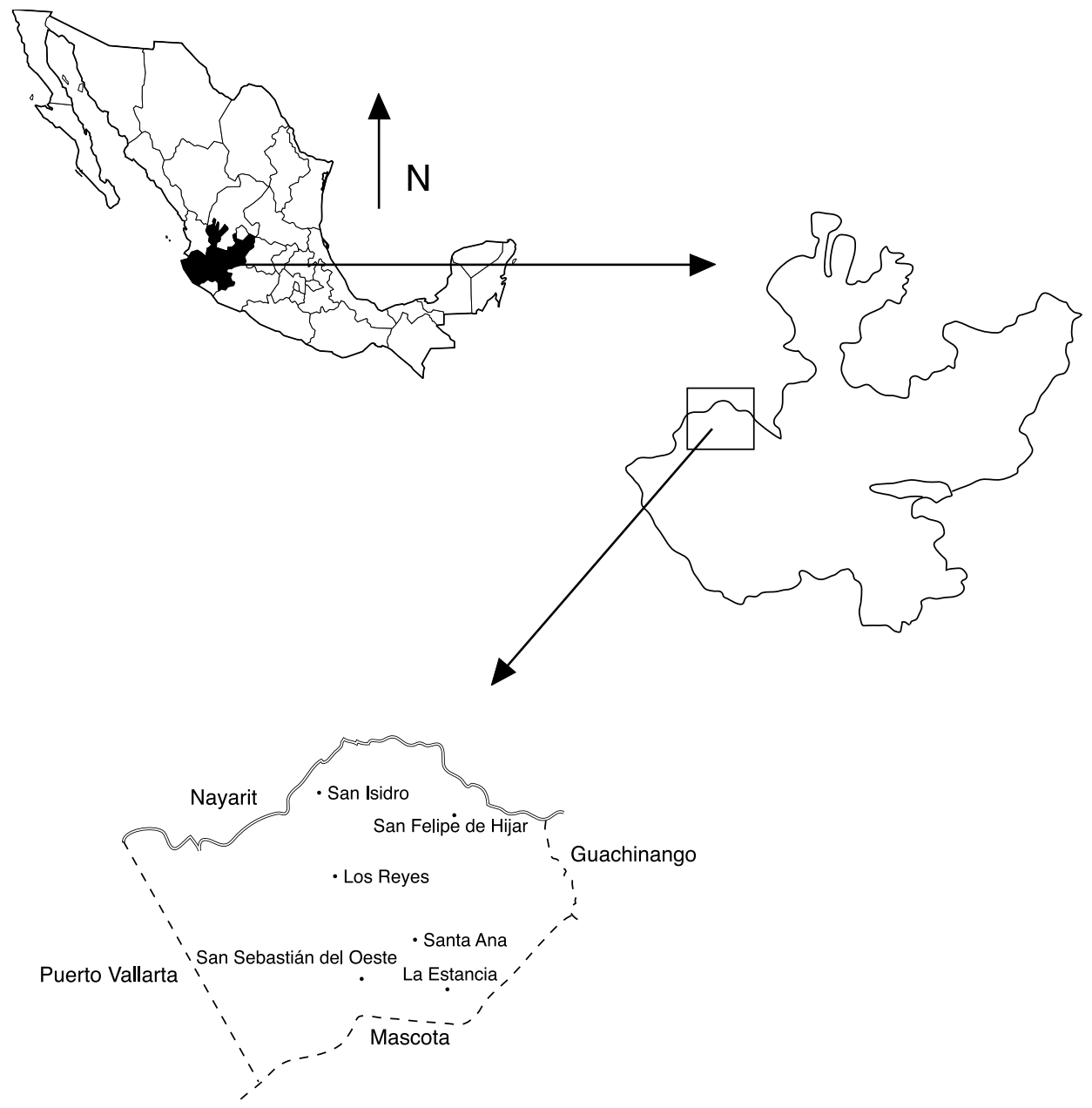

Fig. 1. Localización del municipio de San Sebastián del Oeste, Jalisco.

de Melzer y Rojo Congo. La identificación se efectuó principalmente con las claves de Guzmán (1977) y después con bibliografía especializada, como la de Breitenbach y Kränzlin (1986), Coker (1974), Dennis (1970), Gilbertson y Ryvarden (1986, 1987), Petersen (1988), entre otras. Algunos especímenes se compararon con los de otras regiones, provenientes de los herbarios FCME, IBUG y MEXU y de los particulares de Rodham Tulloss (E.U.A.) y Francis Massart (Francia). Para apoyar la identificación de los ejemplares del orden 
Poriales se revisó y comparó el material depositado en el herbario IBUG, el cual había sido determinado por Ricardo Valenzuela en 1997, especialista en este grupo. Varios especímenes de Poriales fueron identificados directamente por Valenzuela.

\section{RESULTADOS Y DISCUSIÓN}

\section{Material fúngico determinado}

De los 1060 ejemplares, procedentes del municipio de San Sebastián del Oeste, se lograron identificar 583, que corresponden a 216 especies. A diferencia de otros inventarios efectuados en Jalisco, como los de Rodríguez et al. (1994) y Fierros y Guzmán-Dávalos (1995), el número de determinaciones en este trabajo superó el total de aquéllos (103 especies del Volcán de Tequila y 159 de la Sierra de Quila, respectivamente). El apéndice presenta el listado de los hongos estudiados, donde se señalan los 35 nuevos registros para el estado de Jalisco y dos para la micobiota mexicana: Amanita roseitincta y Ramaria abietina. En este apéndice se siguió el sistema de clasificación de Hawksworth et al. (1995), con modificaciones para los Agaricales según Singer (1986). De las 216 especies 89.4\% corresponden a Basidiomycota, 8.8\% a Ascomycota, 1.3\% pertenece al grupo Myxomycota, y $0.5 \%$ a Deuteromycota. Se incluyeron dos hongos microscópicos (Cronartium conigenum y Sepedonium chrysospermum), el primero porque parasita hongos macroscópicos, del grupo de los Boletáceos y el segundo por desarrollarse a expensas de conos de los pinos.

Del material identificado el orden de los Agaricales fue el más diverso, con 78 especies, y las familias mejor representadas fueron Tricholomataceae con 21 especies, Amanitaceae con 15, Russulaceae con 11, Boletaceae con 9 y Strophariaceae con 8. Por otra parte, en el orden de los Poriales se registran 44 especies y de ellas la familia Coriolaceae, con 32 especies fue la más importante. En cuanto a la distribución ecológica de las especies indicadas en el Apéndice, se encontraron 109 que se adscriben al bosque de pino-encino, 89 al mesófilo de montaña, 39 al de pino, 29 al tropical caducifolio, 14 al tropical subcaducifolio, 13 al de encino, 10 al de encino-pino y 10 a la vegetación secundaria o áreas perturbadas. Estos resultados se pueden comparar con lo presentado por Fierros y Guzmán-Dávalos (1995), en particular con los hongos recolectados en el bosque mesófilo de montaña, quienes registraron 91 especies (57\%) en mesófilo, cifra en proporción más alta a la obtenida aquí (89 especies, 41\%). Chacón y Medel (1993) consideraron 594 especies del bosque mesófilo de montaña de México, 33 de ellas de Jalisco. La cantidad de hongos del bosque mesófilo de montaña que registramos aquí para San Sebastián es baja comparándola con los datos de Fierros y colaboradores (op. cit.) y los de Chacón y Medel (op. cit.). Este resultado refleja la falta de exploraciones micológicas en este tipo de vegetación en la zona, ya que por las condiciones de la región se espera una diversidad alta.

De acuerdo con el tipo de sustrato (Fig. 2), se encontraron 124 (56\%) especies lignícolas, $83(37 \%)$ terrícolas, $11(5 \%)$ humícolas, 2 (1\%) fungícolas, una fimícola, una entomopatógena, y una más fitopatógena. A diferencia de otros inventarios, como los de Cifuentes et al. (1990), Rodríguez et al. (1994) y Fierros y Guzmán-Dávalos (1995), el número de elementos lignícolas resultó alto en comparación con la cantidad de especies 
citadas por los autores mencionados. Esto puede ser debido a que en los bosques tropicales se desarrollan en su mayoría hongos lignícolas, principalmente por el clima y la humedad que favorecen la rápida descomposición de la materia orgánica (Guzmán-Dávalos y Guzmán, 1979). También el gran número de especies lignícolas probablemente esté influenciado por un mayor esfuerzo de identificación en los Poriales, comparado con el de los trabajos mencionados. En cuanto a la importancia de las especies estudiadas (ver Fig. 3), 113 (43\%) son destructoras de madera, 70 (27\%) micorrízicas, 42 (16\%) comestibles, $21(8 \%)$ venenosas, 10 (4\%) parásitas de hongos, insectos o plantas, y $4(2 \%)$ son alucinógenas.

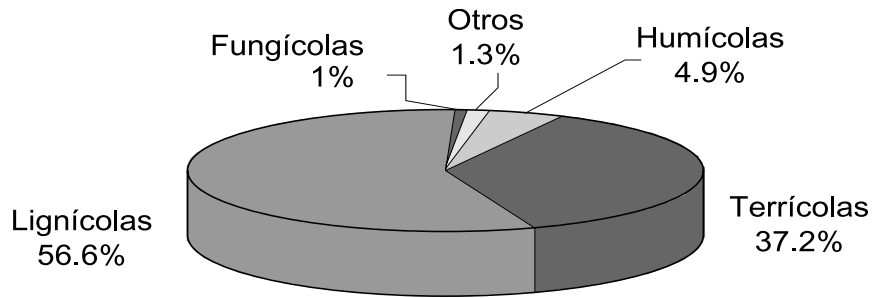

Fig. 2. Distribución de las especies por tipo de sustrato. Otros incluyen las categorías de fimícola, entomopatógena, y fitopatógena, cada uno con una especie.

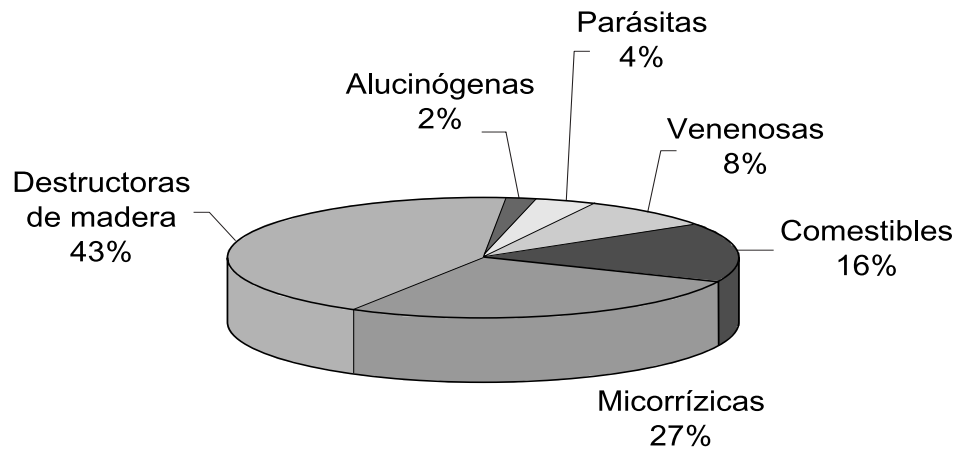

Fig. 3. Importancia económica y ecológica de las especies. 
A continuación se incluyen observaciones y comentarios de los nuevos registros de Jalisco. En el caso de los propios para México, se describe completamente a Ramaria abietina; para Amanita roseitincta únicamente se hacen comentarios, ya que su descripción será presentada en un trabajo posterior.

\section{Nuevos registros de Jalisco}

Boletellus betula (Schwein.) Gilb.

Basidioma con píleo amarillo-canario a amarillo-mostaza o amarillo-limón y hacia el margen de color rojizo, con la superficie viscosa, brillante y lisa. Himenóforo con poros hexagonales, adheridos o subdecurrentes, de color amarillo-canario. Estípite reticulado, finamente lanoso hacia el centro, concoloro con el himenóforo, manchado irregularmente de color rojo-ladrillo.

Solamente se conocía de Durango e Hidalgo, según Bandala-Muñoz et al. (1988) y de Guerrero, Estado de México y Tamaulipas, según Singer et al. (1992).

Material estudiado. La Bufa, alt. 2300 m, julio 24, 1998, M. Herrera 265.

Calocera cornea (Bastch : Fr.) Fr.

Figs. 4-6

El material estudiado concuerda con lo descrito por Breitenbach y Kränzlin (1986). Se caracteriza por presentar un basidioma claviforme, simple o bifurcado, de consistencia cartilaginosa a gelatinosa, amarillo en el ápice y anaranjado hacia la base. Las esporas son de 8-9.6 x 3.6-4.4 $\mu \mathrm{m}$, alargado-cilíndricas, con un septo transversal, de pared delgada, hialinas. Los basidios son bifurcados, con gotas de contenido, de pared delgada y hialinos. Las hifas son generativas, septadas, de pared delgada-subgruesa.

Material estudiado. Entronque camino San Sebastián del Oeste, La Mesa y Cieneguitas, alt. 1000 m, julio 13, 1997, L. Guzmán-Dávalos 6510, O. Rodríguez 1652.

Clavaria aff. fistulosa Fr.

Figs. 7-10

Basidioma de 50-120 x 2-3 $\mu \mathrm{m}$, claviforme, simple, con terminación en punta de color café-rojizo, el resto es amarillo-anaranjado. Esporas de 9.6-11.2 x 8.4-11.2 $\mu \mathrm{m}$, globosas, de pared delgada, hialinas. Basidios claviformes con 2 esterigmas, rara vez 3 , ocasionalmente con un septo transversal. Hifas del contexto de pared delgada, hialinas, con fíbulas poco frecuentes.

De acuerdo con Petersen (1988), el espécimen pertenece al género Clavaria, subgénero Clavulinopsis por presentar fíbulas. Las características macroscópicas son similares a las de Clavariadelphus fistulosus (Fr.) Corner (= Clavaria fistulosa Fr.). Sin 
embargo, de acuerdo con Phillips (1981), microscópicamente la forma y tamaño de las esporas es muy diferente; pues éstas son subfusiformes y miden 10-18.5 x 4.5-8 $\mu \mathrm{m}$. El material estudiado puede representar una nueva especie, la cual se tiene en estudio.

Material estudiado. Brecha San Sebastián del Oeste-La Bufa, cerca del Segundo Arroyo, alt. 1300-1400 m, septiembre 14, 1995, O. Rodríguez 1338.

\section{Clavulinopsis corniculata (Fr.) Corner}

Basidioma claviforme y ramificado hacia el ápice, con 3-4 ramificaciones cortas, lisas, delgadas, redondeadas. Superficie amarillo-rosada, puntas manchadas de rojizo y base blanquecina. La reacción con $\mathrm{KOH}$ es positiva, revelando color anaranjado en la superficie del basidioma. Esporas de 6.4-8 x 6.4-8.4 $\mu \mathrm{m}$, globosas, lisas, de pared delgada, hialinas.

El material concuerda con lo descrito por Coker (1974), quien la identificó como Clavaria muscoides Lowy. La especie aquí estudiada sólo se conocía de los estados de Hidalgo y Morelos (Bandala-Muñoz et al., 1987b).

Material estudiado. Km 6 brecha San Sebastián del Oeste-El Real Alto, Portezuelo de Santa Ana, alt. 1900 m, septiembre 14, 1995, M. R. Sánchez-Jácome 857.

\section{Coltriciella pseudocinnamomea Burds}

Se caracteriza microscópicamente por presentar las esporas ornamentadas, con verrugas finas, carácter de importancia, ya que la separa del género Coltricia que tiene esporas lisas. El material revisado concuerda con lo descrito por Gilbertson y Ryvarden (1986).

Material estudiado. Entronque camino a San Sebastián del Oeste, La Mesa y Cieneguitas, alt. 1000 m, julio 13, 1997, L. Guzmán-Dávalos 6505.

\section{Cookeina venezuelae (Berk. \& Curtis) Le Gal}

Figs. 11-12

Ascoma de 10-20 mm de diámetro, en forma de copa, cartilaginoso, de color anaranjado-rosado. Estípite corto o muy corto, blanco, lacunoso con oquedades a todo lo largo. Esporas de 33.6-37.2(-38.4) x 12-13.2 $\mu \mathrm{m}$, alargadas a cilíndricas, ornamentadas con 4 líneas longitudinales gruesas.

El material revisado se determinó de acuerdo con la descripción de Dennis (1970), quien indicó que las esporas miden 30-40 x 12-15 $\mu \mathrm{m}$ y llevan 4-6 líneas longitudinales. C. venezuelae fue registrada por Guzmán (1977) sin localidad precisa y por Frutis y Guzmán (1983) del estado de Hidalgo.

Material estudiado. Brecha Milpillas-La Virgencita, Cañada Milpillas, alt. 13001400 m, agosto 13, 1998, L. Guzmán-Dávalos 7326; Segundo Arroyo-El Real Alto, alt. 1400- 
1460 m, agosto 14, 1998, M. Herrera 289, O. Rodríguez 1884, octubre 8, 1998, M. Herrera 569.

Coriolopsis brunneoleuca (Berk.) Ryvarden

Especie poco común para el área de estudio, hasta ahora citada de los estados de Hidalgo, Tamaulipas y Michoacán, según Bandala-Muñoz y colaboradores (1993).

Material estudiado. La Haciendita (San Isidro), alt. 470-550 m, agosto 16, 1998, M. Herrera 387.

Coriolopsis byrsina (Mont.) Ryvarden

Su basidioma es efuso-reflejo, delgado, con una superficie tomentosa-pubescente, de color café-mostaza y el himenóforo formado por poros hexagonales, pequeños, de color crema a concoloros con el píleo. Se registra por primera vez de Jalisco, siendo poco frecuente en la zona de estudio.

Material estudiado. Arroyo Zonteco, km 21 brecha San Sebastián del Oeste-La Haciendita (San Isidro), alt. 500 m, agosto 16, 1998, O. Rodríguez 1941.

Dacryopinax elegans (Berk. \& Curtis) G. W. Martin

Figs. 13-15

Basidioma cocleriforme a espatulado, cartilaginoso-gelatinoso, de color café-rojizo hacia el centro y café-anaranjado hacia el margen. Esporas de 13.6-15.6(-16) x 5.6-6.4 $\mu \mathrm{m}$, alargadas a cilíndricas, con 1-3 septos transversales, de pared delgada, hialinas. Basidios bifurcados, claviformes a cilíndricos. Hifas de pared gruesa, hialinas, septadas, sin fíbulas.

Se conocía de Veracruz (Welden y Guzmán, 1978), Michoacán y Nayarit (Sierra y Cifuentes, 1993).

Material estudiado. Arroyo El Viborón, brecha San Sebastián del Oeste-San Felipe de Hijar, alt. 750 m, agosto 15, 1998, L. Guzmán-Dávalos 7364, M. Herrera 328.

Datronia mollis (Sommerf. : Fr.) Donk

Basidioma característico por presentar un crecimiento resupinado, con la superficie pubescente de color café-avellana a café obscuro, con el margen delgado y lobulado; su himenóforo está compuesto por poros circulares a angulares, dentados, con 1-2 poros por milímetro. Según Ojeda-López et al. (1986), Datronia esteroides (Fr.) Ryvarden es una 
especie afín, que se diferencia por el tamaño de los poros más pequeños (4-5 por milímetro) y de forma regular.

Anteriormente se conocía de Baja California Sur, Chihuahua, Durango, Guanajuato, Sinaloa, Tamaulipas y Veracruz (Bandala-Muñoz et al.,1993).

Material estudiado. Camino San Sebastián del Oeste-La Bufa, Segundo Arroyo, alt. 1320 m, junio 27, 1998, M. Herrera 169 B.

Dictyopanus pusillus var. rhiphidium (Berk.) Singer

Variedad caracterizada por presentar el basidioma pequeño no mayor de $1 \mathrm{~cm}$ de diámetro, con estípite lateral y corto, blanquecino a amarillento y que cambia una vez seco a color café-rojizo.

Material estudiado. Brecha San Sebastián del Oeste-La Bufa, cerca del Segundo Arroyo, alt. 1490-1630 m, septiembre 14, 1995, O. Rodríguez 1367 A; cerro entre La Taberna de la Ermita y El Otatal, alt. 1250 m, septiembre 16, 1995, L. Guzmán-Dávalos 6040; km 85 brecha Mascota-San Felipe de Hijar, La Uña de Gato, alt. 1330 m, agosto 15, 1998, L. Guzmán-Dávalos 7347, O. Rodríguez 193; La Haciendita (San Isidro), alt. 470-550 m, agosto 16, 1998, M. Herrera 366, 388, O. Rodríguez 1953.

\section{Elaphomyces granulatus Fr.}

Ascomiceto hipogeo frecuentemente parasitado por Cordyceps capitata (Holmsk. : Fr.) Link, de consistencia semicarnosa a dura, de color obscuro con granulaciones llamativas de color amarillo en la superficie externa.

Material estudiado. Km 22 brecha Mascota-San Sebastián del Oeste, La Virgencita, alt. 1850 m, septiembre 11, 1987, L. Guzmán-Dávalos 4039.

Fomitopsis feei (Fr.) Kreisel

Se caracteriza por presentar un basidioma dimidiado, con el píleo pubescente, de color rosa con tonos de color café. El himenóforo está formado por poros muy pequeños concoloros con el píleo, pero con tonos violáceos, hacia la base en ejemplares jóvenes, y en todo el himenio en especímenes maduros.

Presenta una distribución bastante amplia, según datos obtenidos de Bandala-Muñoz et al. (1993), quienes la citaron de 12 estados de México.

Material estudiado. Camino San Sebastián del Oeste-La Bufa, Segundo Arroyo, alt. 1320 m, junio 27, 1998, M. Herrera 179, octubre 8, 1998, M. Herrera 546. 
Gymnopus alkalivirens (Singer) Halling

Especie que se distingue por el píleo de color café-amarillento a café-rojizo-negruzco, con superficie lisa. Las láminas son subadheridas, de color café-grisáceo a café-rojizo o café-negruzco y el estípite es fibriloso de color café obscuro o negro.

Se conoce del Estado de México de acuerdo con Villarruel-Ordaz et al. (1993).

Material estudiado. El Llanito de Hielos-La Bufa, alt. 2160 m, agosto 28, 1994, O. Rodríguez 1035.

Hymenochaete sallei Berk. \& Curtis

Es una especie muy abundante en la zona de estudio, caracterizada por su píleo flabeliforme, zonado, velutino a tomentoso y por la fragilidad del mismo, además por la línea de color amarillo-mostaza que presenta en el margen. El resto de la superficie del basidioma es de color café-ferruginoso pasando a café obscuro hacia la base.

Material estudiado. Brecha San Sebastián del Oeste-La Bufa, cerca del Segundo Arroyo, alt. 1490 m, septiembre 14, 1995, O. Rodríguez 1363, octubre 8, 1998, M. Herrera 554, 567; brecha a Los Reyes, al E de San Sebastián del Oeste, junio 26, 1998, M . Herrera 109, 117; km 85 brecha Mascota-San Felipe de Hijar, La Uña de Gato, alt. 1330 m, agosto 15, 1998, L. Guzmán-Dávalos 7354, M. Herrera 350, O. Rodríguez 1929; Segundo ArroyoEl Real Alto, octubre 8, 1998, M. Herrera 542.

Hypoxylon monticulosum Mont.

Especie conocida de Chiapas, Michoacán, Nuevo León, Oaxaca, Quintana Roo, Tabasco, Tamaulipas (Medel et al., 1999; San Martín et al., 1999). Se caracteriza por tener un estroma efuso, pulvinado-aplanado, menor de $5 \mathrm{~mm}$ de altura, de consistencia carbonosa y de color negro-violáceo.

Material estudiado. Brecha San Sebastián del Oeste-San Felipe de Hijar, Arroyo El Viborón alt. 750 m, agosto 15, 1998, L. Guzmán-Dávalos 7366.

Inonotus fruticum (Berk. \& Curtis) Murrill

Se caracteriza por la superficie del píleo aterciopelada de color café-amarillentomostaza y por crecer sobre ramas de Annona. Guzmán (1977) la citó del país sin localidad precisa, Bandala-Muñoz y colaboradores (1993) la registraron de los estados de Tamaulipas y Yucatán.

Material estudiado. Brecha San Sebastián del Oeste-San Felipe de Hijar, Arroyo El Viborón, alt. 750 m, agosto 15, 1998, M. Herrera 335. 
Herrera et al.: Contribución al Conocimiento de la Micobiota de San Sebastián del Oeste, Jalisco

Inonotus fulvomelleus Murrill

Las características macroscópicas del material estudiado concuerdan con las dadas por Murrill (1973), quien describió el píleo con la superficie hirsuta, el margen grueso, híspido y el himenóforo compuesto por poros pequeños, circulares, de color amarillo-mostaza.

Material estudiado. Brecha San Sebastián del Oeste-La Bufa, cerca del Segundo Arroyo, alt. 1300-1400 m, agosto 29, 1994, O. Rodríguez 1061; La Bufa, alt. 2350 m, julio 24, 1998, M. Herrera 250.

Marasmius spegazzinii Sacc. \& Syd.

Especie caracterizada por su píleo de color café-anaranjado o café-ferruginoso, con láminas blanquecinas a amarillentas y el estípite blanquecino, con tonos de color café-rojizo en la base. Este es el primer registro de Jalisco, anteriormente se conocía de Durango, Veracruz (Bandala-Muñoz et al., 1988), Michoacán (Díaz-Barriga et al.,1988), Estado de México y Morelos (Chacón y Medel, 1993).

Material estudiado. Brecha de San Sebastián del Oeste-La Bufa, cerca del Segundo Arroyo, alt. 1300-1400 m, agosto 29, 1994, O. Rodríguez 1053; brecha San Sebastián del Oeste-La Bufa, entre Portezuelo de Santa Ana y El Llanito, alt. 1930-2290 m, septiembre 14, 1995, S. Y. Rubio 214.

Nigroporus vinosus (Berk.) Murrill

Especie macroscópicamente fácil de distinguir por el tamaño y el color de su basidioma, de 24-28 mm de diámetro, dimidiado, con el píleo escabroso, de color rosadovioláceo, al igual que el himenóforo, pero éste un poco más tenue, con el borde grisáceo. La reacción en el píleo con $\mathrm{KOH}$ es positiva, de color café obscuro. Su sistema hifal dimítico, la coloración café-rosada de sus hifas esqueléticas y sus esporas cilíndricas a alantoides, son caracteres de valor diagnóstico que la separan de Nigrofomes melanopus (Mont.) Murrill (Gilbertson y Ryvarden, 1987).

Se conocía sólo de los estados de Hidalgo, Tamaulipas y Veracruz, de acuerdo con Bandala-Muñoz et al. (1993).

Material estudiado. Brecha San Sebastián del Oeste-La Bufa, cerca del Segundo Arroyo, alt. 1490-1630 m, septiembre 14, 1995, O. Rodríguez 1353.

Oligoporus caesius (Schrad. : Fr.) Gilb. \& Ryvarden

Es una especie poco común en el área de estudio, que se caracteriza por su basidioma dimidiado, blanquecino, que se mancha irregularmente de azul, carácter de 
importancia, ya que la distingue de O. perdelicatus (Murrill) Gilb. \& Ryvarden, que presenta un basidioma totalmente blanco (Gilbertson y Ryvarden, 1987).

El material estudiado concuerda con lo descrito por Gilbertson y Ryvarden (1987); se hizo además la revisión de ejemplares depositados en el herbario IBUG procedentes de otros municipios de Jalisco, los cuales fueron determinados por Valenzuela en 1997.

Material estudiado. Brecha San Sebastián del Oeste-La Bufa, entre Portezuelo de Santa Ana y El Llanito, alt. 1930-2290 m, septiembre 14, 1995, L. Guzmán-Dávalos 5993.

Otros materiales. Municipio de Cuautitlán, Predio Las Joyas, Sierra de Manantlán, Cuatro Caminos, rumbo al Charco de los Perros, alt. 2000 m, septiembre 28, 1995, S. Y. Rubio 306; municipio de Mezquitic, entre Huejuquilla El Alto y Los Amoles, alt. 2500 m, julio 31, 1996, O. Rodríguez 1457; municipio de Tapalpa, km 2 camino a Tapalpa, 2135 m, agosto 14, 1995, O. Rodríguez 1242; municipio de Tecalitlán, Sierra del Halo, km 27 brecha Tecalitlán-Jilotlán, alt. 2100 m, agosto 22, 1985, L. Guzmán-Dávalos 2307.

\section{Oligoporus floriformis (Quél.) Gilb. \& Ryvarden}

Se caracteriza principalmente por la fructificación lateralmente subestipitada y por la coloración del píleo, la cual es blanquecina a grisácea. Según Gilbertson y Ryvarden (1986), macroscópicamente es similar a Antrodiella semisupina (Berk. \& Curtis) Ryvarden, la cual tiene un sistema hifal trimítico y esporas elipsoides, a diferencia de 0 . floriformis, que presenta un sistema hifal monomítico y esporas oblongas a cortamente cilíndricas, ligeramente curvadas.

La especie aquí citada se conocía de los estados de Hidalgo y Veracruz (BandalaMuñoz et al., 1993).

Material estudiado. Portezuelo de Santa Ana, aproximadamente a $6 \mathrm{~km}$ de San Sebastián del Oeste, hacia El Real Alto, alt. 1900 m, septiembre 14, 1995, L. GuzmánDávalos 5928.

\section{Phellinus sarcites (Fr.) Ryvarden}

Su basidioma es flabeliforme, caracterizado por presentar un color anaranjado-café en la base y amarillo-mostaza hacia el margen, el cual es ligeramente lobulado y delgado. La superficie del píleo es hirsuta, estriada con una apariencia de bordes o pliegues levantados. El himenóforo es amarillo-dorado hacia el borde y de color café obscuro con tonos mostaza hacia la base.

Se amplía la distribución conocida de esta especie al citarla del estado de Jalisco, ya que anteriormente había sido registrada de Morelos, Oaxaca y Veracruz (Bandala-Muñoz et al., 1993).

Material estudiado. Brecha a Los Reyes al E de San Sebastián del Oeste, junio 26, 1998, M. Herrera 114. 
Otros materiales. Municipio de Zapopan, parque Los Colomos II, noviembre 19, 1993, M. Gutiérrez 31; municipio de Tequila, brecha a La Estación de Microondas en el cerro de Tequila, alt. 2450-2500 m, agosto 9, 1996, O. Rodríguez 1493; municipio de Zapotlán el Grande, faldas del Nevado de Colima, El Floripondio, alt. 2000-2150 m, septiembre 7, 1996, O. Rodríguez 1610.

Phillipsia aff. domingensis (Berk.) Berk.

Figs. 16-17

El ascoma es en forma de disco subcóncavo, con superficie aterciopelada, brillante, de color tinto-guinda con tonos violáceos. El estípite es corto y mal definido, blanquecino. Esporas de 26.4-28 x 12-13.6 $\mu \mathrm{m}$, alargadas, ornamentadas por numerosas líneas o estrías longitudinales, de pared gruesa, hialinas.

Por las características macroscópicas que presenta, se puede pensar que se trata de P. domingensis (Berk.) Berk., de acuerdo con Guzmán (1977); sin embargo, microscópicamente la ornamentación de las esporas observadas es diferente de la ofrecida en la descripción de Dennis (1970), en la que se definen 4-6 líneas longitudinales, mientras que en el ejemplar estudiado son más de seis.

Por otra parte, se revisó la descripción de Bautista et al. (1986), quienes para $P$. domingensis mencionaron esporas alargadas, estriadas, pero no indicaron la cantidad de estrías. El resto de las características concuerda con las que se observaron en el ejemplar estudiado.

Material estudiado. Segundo Arroyo-El Real Alto, alt. 1400 m, agosto 14, 1998, M. Herrera 290; brecha San Sebastián del Oeste-San Felipe de Hijar, Arroyo El Viborón, alt. 750 m, agosto 15, 1998, L. Guzmán-Dávalos 7380; km 21 brecha San Sebastián del OesteLa Haciendita (San Isidro), Arroyo Zonteco, alt. 470-550 m, agosto 16, 1998, L. GuzmánDávalos 7427, M. Herrera 356; km 2 brecha Mascota-San Felipe de Hijar, antes de La Uña de Gato, alt. 500 m, agosto 16, 1998, L. Guzmán-Dávalos 7385.

\section{Pholiota rigidipes Peck}

Se caracteriza por presentar el píleo escamoso, de color café con tonos mostaza, las láminas adheridas al estípite, el cual es escamoso-lanoso hacia la base. Sólo se conocía de los estados de México y Morelos (Bandala-Muñoz et al.,1988).

Material estudiado. El Llanito de Hielos, brecha San Sebastián del Oeste-La Bufa, alt. 2160 m, agosto 28, 1994, O. Rodríguez 1032, 1034.

Ramaria araiospora var. rubella Marr \& D. E. Stuntz Figs. 18-20

El basidioma es coraloide, con ramificaciones dicotómicas y otras secundarias hacia el ápice, superficie fibrilosa de color rojo-fresa brillante y con la base blanquecina. La 


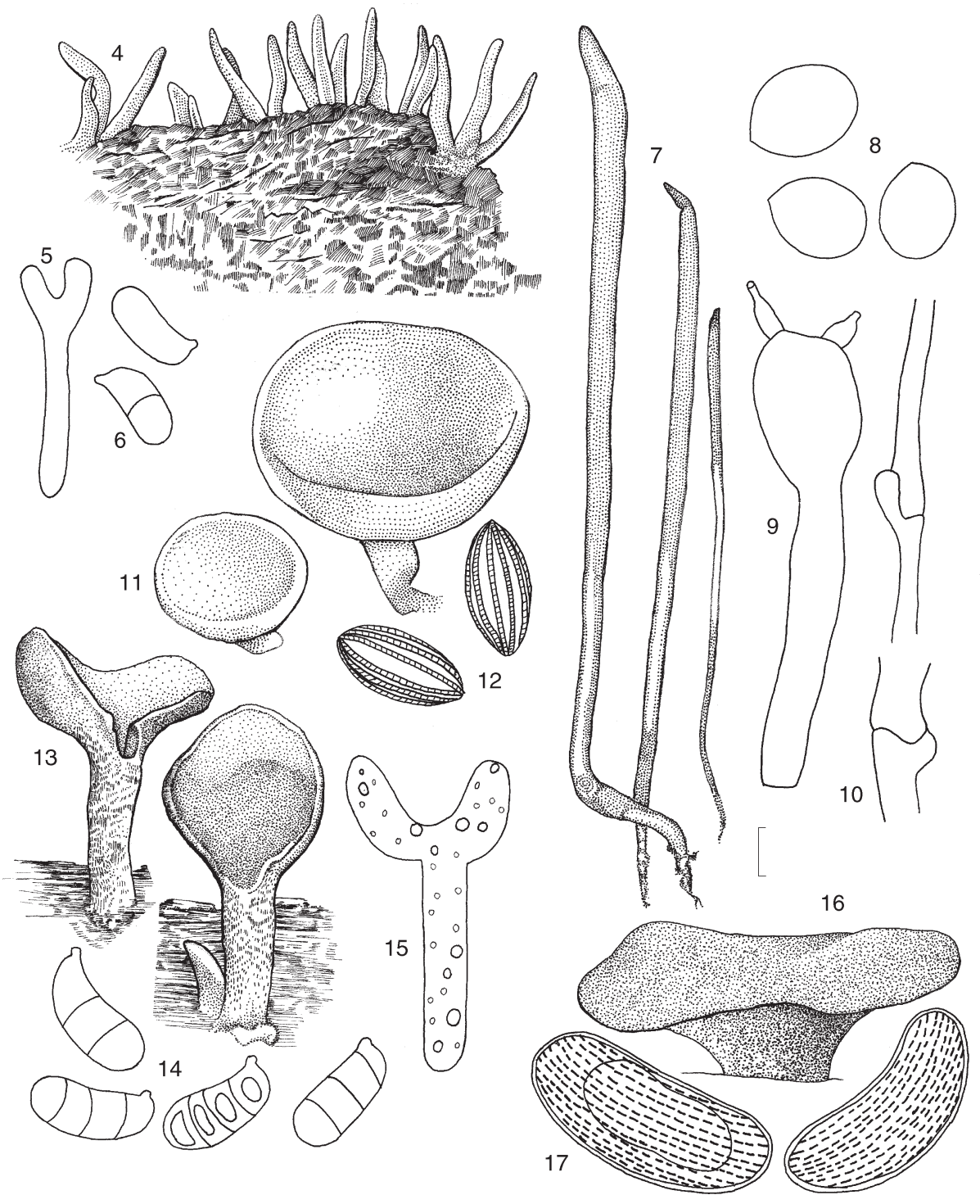

Figs. 4-17. 4-6: Calocera cornea, 4: basidiomas, 5: basidio, 6: esporas. 7-10: Clavaria aff. fistulosa, 7: basidiomas, 8: esporas, 9: basidio, 10: hifas del contexto. 11-12: Cookeina venezuelae, 11: ascomas, 12: esporas. 13-15: Dacryopinax elegans, 13: basidiomas, 14: esporas, 15: basidio. 16-17: Phillipsia aff. domingensis, 16: ascoma, 17: esporas (escala $=8 \mu \mathrm{m}$, excepto $4=0.5 \mathrm{~cm}, 7,11,13 \mathrm{y}$ $16=0.3 \mathrm{~cm})$. 
reacción con $\mathrm{KOH}$ es positiva en la superficie y de color amarillo-olivo claro. Las esporas son de 9.6-11.6 x 4-4.8 $\mu \mathrm{m}$, subcilíndricas a cilíndricas, verrugosas, de pared delgada, hialinas.

El material de Jalisco concuerda con lo mencionado por Phillips (1991), tanto en las características macroscópicas como microscópicas. Pérez-Ramírez et al. (1986) definieron las esporas como elípticas y ligeramente más pequeñas de (7.7-)8.4-11.2 x 3.5$4.2 \mu \mathrm{m}$ para esta variedad. Es muy afín a $R$. subbotrytis var. intermedia Coker, descrita por Bandala-Muñoz et al. (1987a) con esporas de 9-12 x 3.5-4(-5) $\mu \mathrm{m}$, elipsoides a subfusiformes, rugosas, semejantes al espécimen estudiado, pero macroscópicamente diferente en el color del basidioma, ya que presenta una gama de combinaciones que van desde rosa-rojizo a crema-amarillento con tonos rosados.

Este es el segundo registro que se tiene para la micobiota mexicana de $R$. araiospora var. rubella, ya que sólo se conocía del estado de Michoacán, según Pérez-Ramírez y colaboradores (1986).

Material estudiado. Camino San Sebastián del Oeste-La Bufa, Portezuelo de Santa Ana y El Llanito, alt. 1930 - 2290 m, septiembre 16, 1995, O. Rodríguez 1388.

Rigidoporus microporus (Fr.) Overeem

Su basidioma es dimidiado, con la superficie glabra, zonada concéntricamente, con líneas de color café-anaranjado a café-rojizo, el resto de la superficie es de color paja con tonos ocráceos y su margen es enrollado, delgado. El himenóforo presenta un color rosa con tonos violáceos.

El color del basidioma en fresco y el tamaño de los poros lo distinguen de $R$. lineatus (Pers.) Ryvarden, ya que la coloración que presenta esta especie es más obscura (Gilbertson y Ryvarden, 1987).

Material estudiado. Cañada Milpillas, brecha Milpillas-La Virgencita, alt. 1300-1400 m, agosto 13, 1998, L. Guzmán-Dávalos 7324; km 21 brecha San Sebastián del Oeste-La Haciendita (San Isidro), Arroyo Zonteco, alt. 500 m, agosto 16, 1998, O. Rodríguez 1949.

Sarcosoma mexicanum (Ellis \& Holw.) Paden \& Tylutki

Ascoma en forma de copa, de consistencia elástica-cartilaginosa, liso, con el interior de color café obscuro-negro y el exterior gris-negro. Se registra además del municipio de Mezquitic.

Material estudiado. La Bufa, alrededores de la antena de Telmex, alt. 2300-2400 m, agosto 28, 1994, O. Rodríguez 1036.

Otros materiales. Municipio de Mezquitic, entre Huejuquilla El Alto y Los Amoles, alt. 2500 m, julio 31, 1996, O. Rodríguez 1449, en bosque de Abies durangensis con elementos de Pinus y Quercus. 
Steccherinum ochraceum (Fr.) Gray

Esta especie había sido citada sólo para el estado de Oaxaca (Welden et al., 1979). Es poco común en el área de estudio; se caracteriza por presentar un margen lobulado de color blanquecino a crema, con el resto de la superficie gris-plateada a gris obscura con zonas concéntricas blanquecinas, y el himenóforo formado por dientes de color blanquecino a crema.

Material estudiado. Brecha de Los Reyes al E de San Sebastián del Oeste, junio 26, 1998, M. Herrera 99.

Stereum frustulatum (Pers. : Fr.) Fuckel

El material estudiado concuerda con lo descrito por Marmolejo et al. (1981). Se caracteriza por su basidioma tuberculiforme a resupinado, unido al substrato por una base estrecha. La superficie superior es de color café obscuro a negro, concéntricamente sulcada y el himenóforo liso a granuloso, de color blanquecino a crema. S. frustulatum se conocía de Morelos, Estado de México, Nuevo León, Veracruz y Tamaulipas (Marmolejo et al.,1981).

Material estudiado. La Bufa, 2350 m, julio 24, 1998, M. Herrera 262; camino San Sebastián del Oeste-La Bufa, Segundo Arroyo, alt. 1400 m, agosto 14, 1998, M. Herrera 287, octubre 8, 1998, M. Herrera 552, 562; Cañada Milpillas, brecha-Milpillas-La Virgencita, alt. 1600-1700 m, agosto 11, 1998, M. Herrera 300; alt. 1300-1400 m, agosto 13, 1998, L. Guzmán-Dávalos 7313; km 85 brecha Mascota-San Felipe de Hijar, La Uña de Gato, alt. $1300 \mathrm{~m}$, agosto 15, 1998, M. Herrera 346.

Stereum ochraceoflavum (Schwein.) Ellis

Esta especie se caracteriza por tener un basidioma cupuliforme a efuso-reflejo, con el píleo viloso-hirsuto, ligeramente zonado, blanquecino y con la superficie del himenóforo blanquecina a amarillenta y además por su crecimiento sobre pequeñas ramas de angiospermas.

Se amplía su distribución conocida al registrarla de Jalisco, anteriormente fue citada de Morelos, Estado de México y Veracruz (Marmolejo et al., 1981).

Material estudiado. Brecha San Sebastián del Oeste-La Bufa, Segundo Arroyo, alt. 1320 m, junio 27, 1998, M. Herrera 180; agosto 14, 1998, L. Guzmán-Dávalos 7300.

\section{Stereum subpileatum Berk. \& Curtis}

Basidioma con la superficie del píleo de color café-canela a café obscuro, tomentosa, concéntricamente sulcada y el himenóforo liso a ligeramente agrietado, blanquecino a amarillento o de color crema. 
El material fue comparado macroscópicamente con ejemplares recolectados en los municipios de Cuautitlán y Zapopan depositados en el Herbario IBUG, los cuales habían sido determinados por Valenzuela en 1997. Además los especímenes concuerdan con lo descrito por Marmolejo et al. (1981), quienes la consideraron una especie de amplia distribución.

Material estudiado. Brecha San Sebastián del Oeste-La Bufa, Segundo Arroyo, alt. 1400-1460 m, agosto 14, 1998, M. Herrera 279, O. Rodríguez 1885.

Otros materiales. Municipio de Zapopan, bosque de Los Colomos, junio 28, 1992, Velazco 23; municipio de Cuautitlán, Sierra de Manantlán, Reserva de la Biosfera, Puerto de San Campus, alt. 2010-2100 m, agosto 30, 1995, O. Rodríguez 1272.

Tremella aff. dumontii Lowy

Figs. 21-24

Basidioma gelatinoso, sublaminar-lobado, blanquecino. Esporas de 7.2-9.2 x 7.2$8.8 \mu \mathrm{m}$, globosas a subglobosas, algunas ampliamente-elipsoides, apiculadas, de pared delgada, hialinas. Metabasidios subglobosos, con uno o dos septos longitudinales, de 24 esterigmas, hialinos. Hifas de pared delgada a subgruesa, septadas, con abundantes fíbulas, hialinas.

El material estudiado es afín a T. dumontii Lowy (Lowy, 1980), de acuerdo con la forma y color del basidioma, pero aquel hongo tiene esporas de 8-10.5 x 5.5-8 $\mu \mathrm{m}$, subesférico-ovoides, con un apículo prominente, que no corresponden a las del espécimen de Jalisco. Es probable que se trate de una especie nueva.

Material estudiado. Brecha San Sebastián del Oeste-La Bufa, cerca del Segundo Arroyo, alt. 1300 m, julio 12, 1997, G. Guzmán 32018.

Tremella mesenterica (Retz.) Fr.

Figs. 25-28

Basidioma gelatinoso, cerebriforme, anaranjado-amarillento. Esporas de (8.8-)9.210.4 x 8.8-9.2 $\mu \mathrm{m}$, subglobosas a ampliamente elipsoides, lisas, de pared delgada, hialinas. Metabasidios de 28.4-38.4 x 12.8-13.6 $\mu \mathrm{m}$, subglobosos, con un septo longitudinal; probasidios cilíndricos, tetraspóricos, hialinos. Hifas de pared delgada, hialinas, septadas, con fíbulas.

El material estudiado concuerda con lo descrito por Martin (1969) y Lowy (1971). Macroscópicamente puede confundirse con T. lutescens Fr., que según Martin (op. cit.) presenta basidioma globoso-cerebriforme, de color amarillo claro, pero sus esporas miden 10-16(-20) x 8-12 $\mu \mathrm{m}$. Otra especie afín es Dacrymyces palmatus (Schwein.) Bres., la cual tiene un basidioma petaloide-cerebriforme, anaranjado-rojizo, algo similar al de $T$. mesenterica; sin embargo, sus esporas son cilíndricas con 8 septos transversales, de 23.2$27.2 \times 8.8-10 \mu \mathrm{m}$, según Martin (op. cit.). 


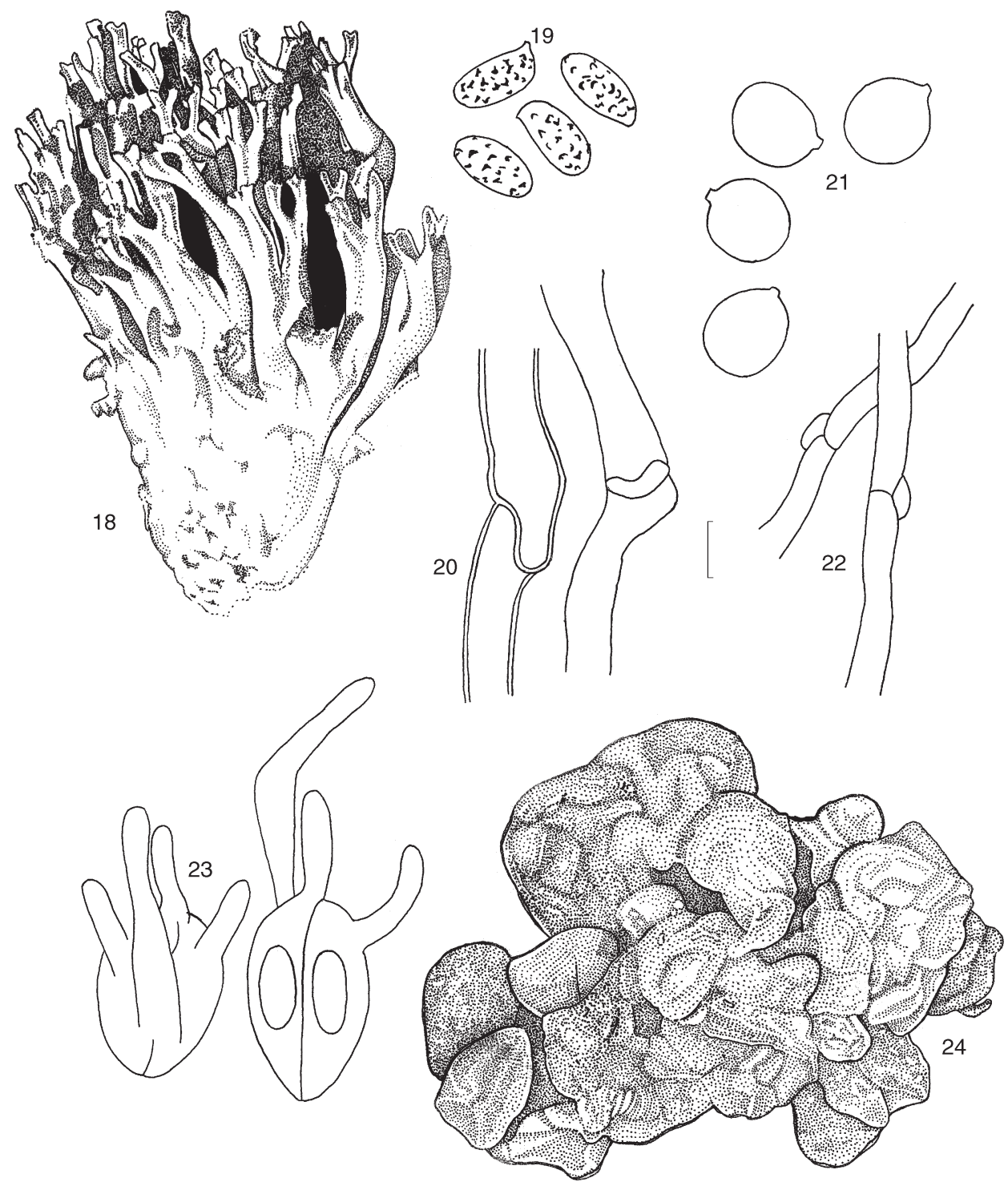

Figs. 18-24. 18-20: Ramaria araiospora var. rubella, 18: basidioma, 19: esporas, 20: hifas del contexto. 21-24: Tremella aff. dumontii, 21: esporas, 22 hifas del contexto, 23: basidios, 24: basidioma (escala $=8 \mu \mathrm{m}$, excepto $18=0.5 \mathrm{~cm}$ y $24=0.2 \mathrm{~cm}$ ). 
Herrera et al.: Contribución al Conocimiento de la Micobiota de San Sebastián del Oeste, Jalisco

Es probable que algunos de los materiales depositados en el Herbario IBUG como $T$. lutescens, estén mal determinados y correspondan a T. mesenterica. En este trabajo se registra por primera vez del estado de Jalisco.

Material estudiado. La Bufa, alt. 2350 m, julio 24, 1998, L. Guzmán-Dávalos 7262.

Trichaptum sector (Ehrenb. : Fr.) P. Karst.

El basidioma es anual y sésil, con el píleo dimidiado a flabeliforme, con superficie tomentosa a velutina, de color paja, zonado con líneas concéntricas de color gris-plateado a café obscuro, y el margen ligeramente lobulado. El himenóforo tiene poros angulares algo dentados, pequeños, de color crema hacia el margen y el resto de la superficie himenófora es de color café obscuro a negro.

Material estudiado. Brecha San Sebastián del Oeste-La Bufa, Segundo Arroyo, alt. 1490 m, septiembre 16, 1995, I. Alvarez 1544.

Nuevos registros de México

Amanita roseitincta (Murrill) Murrill

Su píleo se caracteriza por presentar dos tipos de ornamentación: una superficie pulverulenta-granulosa, que forma escamas planas al agrietarse, de color rosa-salmón, caférosado o ferruginoso, y sobre ella se presentan escamas cónico-truncadas a planas, caedizas, blanquecino-grisáceas. Su estípite es poco bulboso, con fondo blanquecino, pulverulento, que llega a formar escamas pulverulentas, de color rosa-salmón. El anillo es también pulverulento, concoloro con el píleo. La volva es adherida, blanquecina, con un borde o escamas pulverulentas, de color rosa-salmón. Sus esporas son ampliamente elipsoides a elipsoides, en ocasiones globosas y subglobosas. Esta especie es un nuevo registro para México, el cual se describirá en un trabajo posterior (Herrera y GuzmánDávalos, en preparación).

Material estudiado: Brecha San Sebastián del Oeste-La Bufa, cerca del Segundo Arroyo, alt. 1490-1630 m, septiembre 14, 1995, O. Rodríguez 1310.

Ramaria abietina (Pers. : Fr.) Quél.

Figs. 29-31

Basidioma coraloide, de $42 \mathrm{~mm}$ de longitud en seco, con ramificaciones dicotómicas anchas, aplanadas, ramificaciones secundarias delgadas, redondeadas hacia el ápice, base de $8 \mathrm{~mm}$ en seco, velutina a pubescente, superficie de color amarillo-olivo, manchada irregularmente de café-rojizo, con tintes verdosos, base crema-verdosa. Sabor acre y olor agradable. La reacción con $\mathrm{KOH}$ positiva en la superficie, tornándose de color café- 
amarillento a anaranjado. Esporas de 8.4-11.3 x 4-4.8 $\mu \mathrm{m}$, alargadas a cilíndricas, verrugosas, de pared delgada, hialinas. Hifas del contexto de pared delgada, septadas, con fíbulas no muy comunes.

El espécimen estudiado concuerda con los descritos por Petersen (1988) y Breitenbach y Kränzlin (1986) en la presencia de fíbulas en las hifas del contexto y demás características. Se diferencia por presentar tonos verdosos en la base del basidioma y no en las ramificaciones, como lo describen los autores mencionados.

Material estudiado. Brecha San Sebastián del Oeste-La Bufa, cerca del Segundo Arroyo, alt. 1490-1630 m, septiembre 14, 1995, O. Rodríguez 1343.
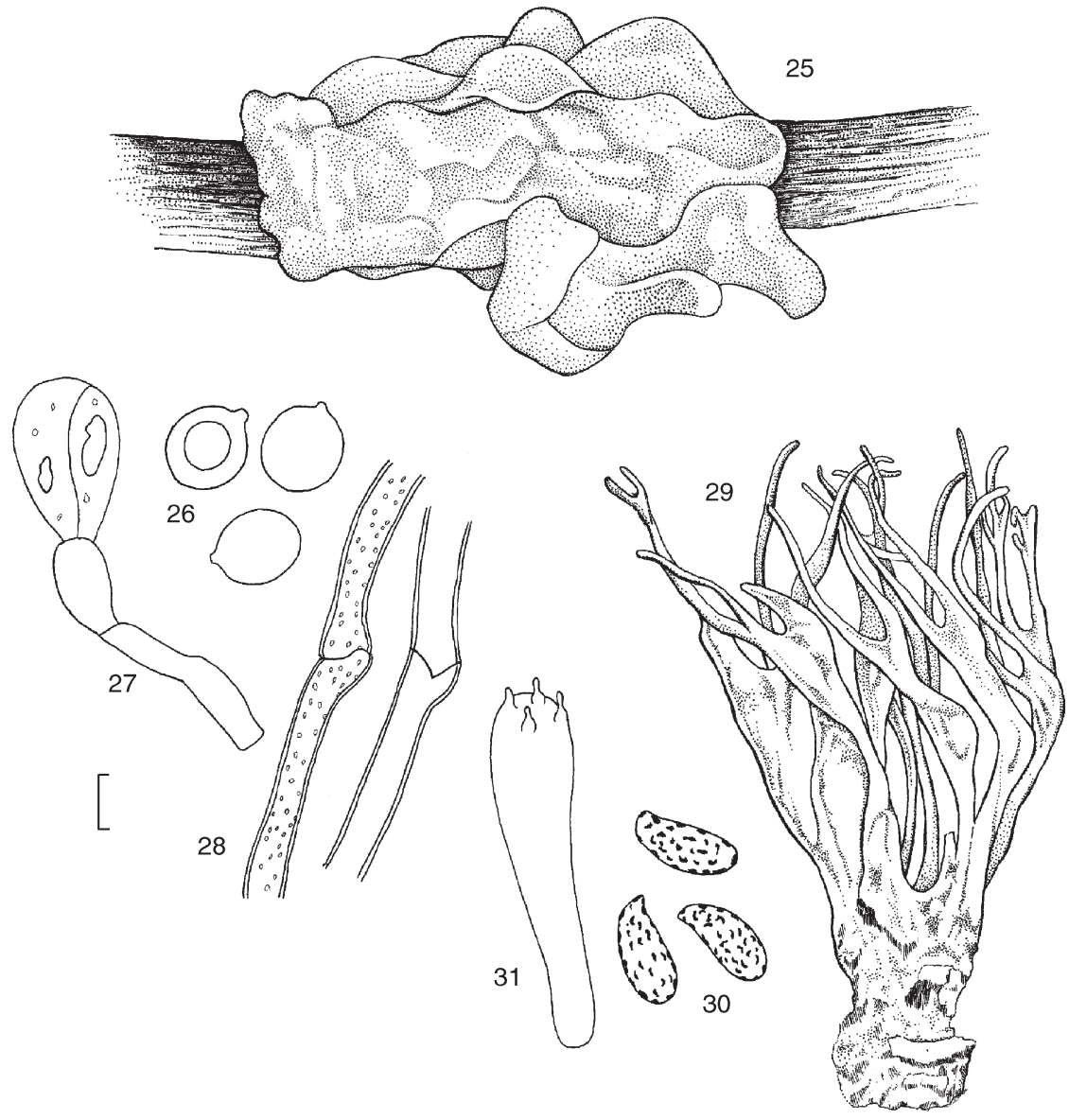

Figs. 25-31. 25-28: Tremella mesenterica, 25: basidioma, 26: esporas, 27: metabasidio, 28: hifas del contexto. 29-31: Ramaria abietina, 29: basidioma, 30: esporas, 31: basidio (escala $=8 \mu \mathrm{m}$, excepto $25=0.2 \mathrm{~cm}$ y $29=0.3 \mathrm{~cm})$. 
Herrera et al.: Contribución al Conocimiento de la Micobiota de San Sebastián del Oeste, Jalisco

\section{APÉNDICE}

Hongos conocidos de la región de San Sebastián del Oeste, Jalisco.

MYXOMYCOTA

CERATIOMYXALES

Ceratiomyxa fructiculosa (Müll. Arg.) T. Macbr. (BTS, BTC, L)

LICEALES

Tubifera ferruginosa (Batsch) J. F. Gmel. (BPE, L)

TRICHIALES

Arcyria incarnata (L.) Wettst. (BMM, BTS, L)

DEUTEROMYCOTA

MONILIALES

Sepedonium chrysospermum Fr. (BMM, Fu, P)

ASCOMYCOTA

ELAPHOMYCETALES

* Elaphomyces granulatus Fr. (BPE, T, M)

HYPOCREALES

Cordyceps capitata (Holmsk. : Fr.) Link (BPE, Fu, P)

C. sphecocephala (Berk.) Sacc. (BTC, I, P)

\section{LEOTIALES}

Leotia lubrica Pers. (BP, BMM, T)

\section{PEZIZALES}

* Cookeina venezuelae (Berk. \& Curtis) Le Gal (BMM, L, DM) Helvella crispa Scop. : Fr. (BPE, T, C, M)

H. elastica Fr. (BPE, T, C, M)

H. lacunosa Fr. (BPE, BMM, T, C, M)

H. macropus (Fr.) P. Karst. (BPE, BMM, T, C, M)

Humaria hemisphaerica (F. H. Wigg.) Fuckel (BMM, T)

* Sarcosoma mexicanum (Ellis \& Holw.) Paden \& Tylutki (BPE, H)

Sarcoscypha coccinea (Scop. : Fr.) Lambotte (BPE, L)

* Phillipsia aff. domingensis (Berk.) Berk. (BMM, BTC, BTS, L, DM)

\section{XYLARIALES}

Daldinia concentrica (Bolt. : Fr.) Ces. \& De Not. (BTC, BTS, L, DM)

D. vernicosa (Schwein.) Ces. \& De Not. (BP, L, DM)

* Hypoxylon monticulosum Mont. (BTS, L, DM) 
Apéndice. Continuación.

H. thouarsianum (Lév.) Lloyd (BPE, L, DM)

Xylaria hypoxylon (L. : Fr.) Grev. (BTC, L, DM)

$X$. polymorpha (Pers. : Fr.) Grev. (BPE, L, DM)

\section{BASIDIOMYCOTA}

BASIDIOMYCETES

CANTHARELLALES

Cantharellaceae

Cantharellus cibarius Fr. (BP, BPE, T, C, M)

C. cinnabarinus (Schwein.) Schwein. (BP, BPE, T, C, M)

C. odoratus (Schwein.) Fr. (BMM, T, C, M)

Clavariaceae

* Clavulinopsis corniculata (Fr.) Corner (BPE, L)

Clavariadelphaceae

* Clavaria aff. fistulosa Fr. (BP, BMM, T, M)

Clavulinaceae

Clavulina rugosa (Fr.) Schröt. (BPE, BMM, H)

Craterellaceae

Craterellus cornucopioides L. : Pers. (BPE, T, C, M)

Hydnaceae

Hydnum repandum $\mathrm{L}$. : Fr. (BP, BPE, T, C, M)

Scutigeraceae

Albatrellus pes-caprae (Pers. : Fr.) Pouzar (BP, L, DM)

\section{GANODERMATALES}

Ganodermataceae

Ganoderma curtisii (Berk.) Murrill (BPE, L, DM, P)

G. Iobatum (Schwein.) G. F. Atk. (BMM, BTC, L, DM, P)

G. Iucidum (Leyss. : Fr.) P. Karst. (BTC, L, DM, P)

\section{GOMPHALES}

Gomphaceae

Gomphus floccosus (Schwein.) Singer (BPE, T, C, M)

Ramariaceae

**Ramaria abietina (Pers. : Fr.) Quél. (BP, BMM, T, M)

${ }^{*}$ R. araiospora var. rubella Marr \& D. E. Stuntz (BP, T, M) 
Apéndice. Continuación.

$R$. aff. lutea (Vittad.) Schild (BMM, T, M)

R. stricta (Fr.) Quél. (BPE, T, M)

\section{HERICIALES}

Auriscalpiaceae

Auriscalpium vulgare S. F. Gray (BPE, L, DM)

Clavicoronaceae

Clavicorona pyxidata (Pers. : Fr.) Doty (BPE, L)

\section{HYMENOCHAETALES}

Hymenochaetaceae

Coltricia cinnamomea (Pers.) Murrill (BPE, L, T)

C. montagnea (Fr.) Murrill (BPE, L, T)

C. perennis (L. : Fr.) Murrill (BP, BPE, L, T)

* Coltriciella pseudocinnamomea Burds (BPE, L, DM)

Hydnochaete tabacina (Berk. \& Curtis) Ryvarden (BPE, L, DM)

* Hymenochaete sallei Berk. \& Curtis (BE, BMM, L, DM)

* Inonotus fruticum (Berk. \& Curtis) Murrill (BTS, L, DM, P)

* I. fulvomelleus Murrill (BP, BPE, BMM, L, DM)

I. radiatus (Sowerby : Fr.) P. Karst. (BPE, L, DM)

Phellinus badius (Berk. ex Cooke) G. H. Cunn. (BPE, L, DM)

P. gilvus (Schwein. : Fr.) Pat. (BPE, L, DM)

P. robustus (P. Karst.) Bourdot \& Galzin (BPE, L, DM)

* $P$. sarcites (Fr.) Ryvarden (BMM, VS, L, DM)

\section{PORIALES}

\section{Coriolaceae}

Bjerkandera adusta (Willd. : Fr.) P. Karst. (BPE, BMM, L, DM)

Climacocystis borealis (Fr.) Kotl. \& Pouzar (BMM, L, DM)

* Coriolopsis brunneoleuca (Berk.) Ryvarden (BMM, BTC, L, DM)

* C. byrsina (Mont.) Ryvarden (BTC, L, DM)

C. polyzona (Pers.) Ryvarden (BTC, L, DM)

* Datronia mollis (Sommerf. : Fr.) Donk (BMM, L, DM)

* Fomitopsis feei (Fr.) Kreisel (BP, BMM, L, DM)

Gloeophyllum sepiarium (Wulfen : Fr.) P. Karst. (BPE, L, DM)

Heterobasidion annosum (Fr.) Bref. (BP, BPE, L, DM)

Hexagonia tenuis (Hook.) Fr. (BMM, L, DM)

H. papyracea Berk. (BTS, L, DM)

Hydnopolyporus fimbriatus (Fr.) D. A. Reid (BPE, BTC, L, T, P)

Lenzites betulinus (L. : Fr.) Fr. (BP, BMM, L, DM)

Megasporoporia mexicana Ryvarden (BPE, L, DM)

* Nigroporus vinosus (Berk.) Murrill (BP, BMM, L, DM) 
Apéndice. Continuación.

* Oligoporus caesius (Schrad. : Fr.) Gillb. \& Ryvarden (BPE, L, DM)

* O. floriformis (Quél.) Gilb. \& Ryvarden (BPE, L, DM)

Phaeolus schweinitzii (Fr.) Pat. (BP, BPE, L, DM)

Pogonomyces hydnoides (Sw. : Fr.) Murrill (BTC, L, DM)

Porodisculus pendulus (Schwein.) Murrill (BPE, L, DM)

Pycnoporus sanguineus (L. : Fr.) Murrill (BE, BTC, L, DM)

* Rigidoporus microporus (Fr.) Overeem (BMM, BTC, L, DM)

Trametes cervina (Schwein. : Fr.) Bres. (BP, L, DM)

T. elegans (Spreng. : Fr.) Fr. (BP, BPE, BTC, VS, L, DM)

T. hirsuta (Wulfen : Fr.) Pilát (BP, L, DM)

T. membranacea (Sw. : Fr.) Kreisel (BTS, VS, L, DM)

T. pavonia (Hook.) Ryvarden (BMM, BTC, VS, L, DM)

T. versicolor (Fr.) Pilát (BP, L, DM)

T. villosa (Fr.) Kreisel (BMM, BTC, VS, L, DM)

Trichaptum abietinum (Dicks. : Fr.) Ryvarden (BPE, L, DM)

T. biforme (Fr.) Ryvarden (BPE, L, DM)

* T. sector (Ehrenb. : Fr.) P. Karst. (BMM, L, DM)

\section{Lentinaceae}

Panus crinitus (L. : Fr.) Singer (BP, BMM, BTC, VS, L, DM)

$P$. badius (Berk.) Singer (BTC, L, DM)

Pleurotus cornucopiae (Paul. : Fr.) Gill. (BPE, L, C, DM)

P. djamor (Fr.) Boedijn (BEP, BTC, L, C, DM)

P. dryinus (Pers. : Fr.) P. Kumm. (BPE, L, C, DM)

$P$. levis (Berk. \& Curtis) Singer (BPE, BMM, L, C, DM)

\section{Polyporaceae}

Polyporus alveolaris (DC. : Fr.) Bondartsev \& Singer (BP, L, DM)

$P$. arcularius Batsch : Fr. (BP, BMM, L, DM)

P. tenuiculus (P. Beauv.) Fr. (BPE, BMM, BTS, VS, L, C, DM)

P. tricholoma Mont. (BP, BMM, BTS, L, DM)

P. tuberaster Jacq. : Fr. (BMM, L, DM)

$P$. varius Fr. (BPE, BMM, L, DM)

\section{SCHIZOPHYLLALES}

Schyzophyllaceae

Schizophyllum commune Fr. (VS, L, C, DM)

S. umbrinum (Berk.) Hook. (BP, BMM, L, DM)

\section{STEREALES}

Meruliaceae

Gloeoporus dichrous (Fr.) Bres. (BPE, L, DM) 
Apéndice. Continuación.

Merulius incarnatus Schwein. (BE, BPE, BMM, L, DM)

M. tremellosus Schrad. : Fr. (BMM, L, DM)

Podoscyphaceae

Cymatoderma caperatum (Berk. \& Mont.) Reid (BTC, L, DM)

Steccherinaceae

* Steccherinum ochraceum (Fr.) Gray (BMM, L, DM)

Irpex lacteus (Fr. : Fr.) Fr. (BPE, BMM, L, DM)

\section{Stereaceae}

Stereum complicatum (Fr.) Fr. (BPE, BMM, L, DM)

* S. frustulatum (Pers. : Fr.) Fuckel (BE, BMM, L, DM)

S. gausapatum (Fr.) Fr. (BE, L, DM)

S. hirsutum (Willd. : Fr.) S. F. Gray (BPE, BMM, L, DM)

* S. ochraceoflavum (Schwein.) Ellis (BMM, L, DM)

S. ostrea (Bull. \& Nees : Fr.) Fr. (BP, BPE, BMM, BTC, L, DM)

* S. subpileatum Berk. \& Curtis (BMM, L, DM)

\section{THELEPHORALES}

Thelephoraceae

Thelephora arbuscula Corner (BEP, T, M)

$T$. vialis Schwein. (BPE, T, M)

\section{AGARICALES}

Hygrophoraceae

Hygrophorus russula (Fr.) Quél. (BEP, T, C, M)

Tricholomataceae

Armillariella mellea (Vahl : Fr.) P. Kumm., s. lat. (BPE, L, C, DM, P)

Clitocybe gibba (Pers. : Fr.) P. Kumm. (BPE, BMM, T, C, M)

Cyptotrama asprata (Berk.) Redhead \& Ginns (BMM, L)

* Dictyopanus pusillus var. rhiphidium (Berk.) Singer (BE, BEP, BTC, L, DM)

* Gymnopus alkalivirens (Singer) Halling (BPE, H)

G. polyphyllus (Peck) Singer (BEP, BMM, H)

Laccaria amethystina (Bolton ex Hook.) Murrill (BP, BPE, T, C, M)

L. laccata (Scop. : Fr.) Berk. \& Bres. (BP, BPE, BMM, T, C, M)

Marasmius guzmanianus Singer (BPE, BMM, H)

$M$. oreades (Bolton : Fr.) Fr. (BE, $\mathrm{H}, \mathrm{C}$ )

M. rotula (Scop. : Fr. ) Fr. (BPE, H, L, DM)

* M. spegazzinii Sacc. \& Syd. (BPE, H, L, DM)

Mycena acicula (Schaeff. : Fr.) P. Kumm. (BMM, H) 
Apéndice. Continuación.

M. leaina (Berk.) Sacc. (BPE, BMM, H, L, DM)

M. pura (Pers. : Fr.) P. Kumm. (BPE, H, C)

Oudemansiella canarii (Jungh.) Höhn. (BPE, BTC, L, C, DM)

Panellus stypticus (Bull. : Fr.) P. Karst. (BPE, L, DM)

Tricholoma flavovirens (Pers. : Fr.) S. Lundell (BMM, T, C)

Tricholomopsis rutilans (Schaeff. : Fr.) Singer (BPE, BMM, T, M)

Xeromphalina campanella (Pers. : Fr.) Kühner \& Maire (BPE, BMM, L, DM)

$X$. tenuipes (Schwein.) A. H. Sm. (BPE, L, DM)

\section{Amanitaceae}

Amanita bisporigera G. F. Atk. (BPE, T, M, V)

A. cokeri (Gilb. \& Kühner) Gilb. (BPE, BMM, T, M, V)

A. flavoconia G. F. Atk. (BPE, BMM, T, M, V)

A. flavorubens (Berk. \& Mont.) Gillb. (BMM, T, M, C)

A. gemmata (Fr.) Gillet (BPE, BMM, T, M, V)

A. magnivelaris Peck (BPE, $\mathrm{T}, \mathrm{M}, \mathrm{V}$ )

A. muscaria var. flavivolvata (Singer) Jenkins (BPE, T, A, M, V)

A. onusta (Howe) Sacc. (BPE, BMM, T, M, V)

A. pantherina (DC. : Fr.) P. Kumm. (BE, T, M, V)

${ }^{*} A$. roseitincta (Murrill) Murrill (BP, BMM, T, M)

A. rubescens (Pers. : Fr.) Gray (BMM, T, C, M)

A. solitaria (Bull. : Fr.) Mérat (BP, BMM, T, M, V)

A. verna (Bull. : Fr.) Roques (BP, T, M, V)

A. vaginata (Bull. : Fr.) Vittad. (BEP, T, C, M)

A. virosa (Fr.) Bertillon (BPE, $\mathrm{T}, \mathrm{M}, \mathrm{V}$ )

Pluteaceae

Pluteus aurantiorugosus (Trog) Sacc. (BMM, L, DM)

P. petasatus (Fr.) Gillet (BTC, L, DM)

\section{Agaricaceae}

Lepiota clypeolaria (Bull. : Fr. ) P. Kumm. (BPE, T)

Leucocoprinus birnbaumii (Corda) Singer (BTC, T)

Coprinaceae

Psathyrella velutina (Fr.) Singer (BPE, T)

Strophariaceae

Hypholoma fasciculare (Huds. : Fr.) P. Kumm. (BPE, L, DM)

Pholiota carbonaria (Fr.) Singer (BPE, L, DM)

* P. rigidipes Peck (BP, BPE, L, DM)

P. spumosa (Fr.) Singer (BPE, L, DM) 
Apéndice. Continuación.

Psilocybe coprophila (Bull. : Fr.) P. Kumm. (BPE, F, V)

$P$. laurae Guzmán (BMM, $\mathrm{T}, \mathrm{A})$

P. subcubensis Guzmán (BE, $\mathrm{T}, \mathrm{A})$

$P$. villarrealiae Guzmán (BMM, $\mathrm{T}, \mathrm{A})$

Cortinariaceae

Inocybe fastigiata (Schaeff. : Fr.) Quél. (BE, T, M, V)

I. geophylla (Sowerby : Fr.) P. Kumm. (BMM, T, M, V)

Crepidotaceae

Crepidotus cinnabarinus Peck (BMM, VS, L, DM)

C. mollis (Schaeff. : Fr.) P. Kumm. (BMM, L, DM)

C. uber (Berk. \& Curtis) Sacc. (BMM, BTS, L, DM)

Paxillaceae

Hygrophoropsis aurantiaca (Wulfen : Fr.) Maire (BMM, T, C, M)

Paxillus panuoides (Fr.) Fr. (BPE, L, DM)

Gomphidiaceae

Gomphidius rutilus S. Lundell \& Nannf. (BPE, T, C, M)

Boletaceae

Boletellus ananas (Curtis) Murrill (BEP, BPE, BMM, T, M)

* B. betula (Schwein.) Gilb. (BPE, T, M) Boletus flammans Dick \& Snell (BPE, T, M) Leccinum aurantiacum (Bull.) Gray (BP, BMM, T, C, M)

Meiorganum curtisii (Berk.) Singer, García \& Gómez (BPE, L, DM)

Strobilomyces confusus Singer (BPE, BMM, T, M)

S. floccopus (Vahl : Fr.) P. Karst. (BMM, T, M)

Tylopilus balloui (Peck) Singer (BPE, T, M)

T. felleus (Bull. : Fr.) P. Karst. (BP, BPE, T, C, M)

Russulaceae

Lactarius deliciosus (L. : Fr.) Gray (BPE, T, C, M)

L. indigo Schwein. : Fr. (BMM, BTS, T, C, M)

L. scrobiculatus (Scop. : Fr.) Fr. (BPE, T, M)

L. vellereus (Fr.) Fr. (BE, T, M)

L. volemus (Fr. ) Fr. (BPE, T, M)

L. piperatus (L. : Fr.) S. F. Gray (BE, T, M)

Russula brevipes Peck (BEP, T, C, M)

R. cyanoxantha (Schaeff.) Fr. (BP, BMM, T, C, M)

$R$. foetens Pers. : Fr. (BPE, T, M, V) 
Apéndice. Continuación.

R. nigricans Bull. : Fr. (BPE, T, C, M)

R. virescens (Schaeff.) Fr. (BPE, T, M, V)

LYCOPERDALES

Lycoperdaceae

Calvatia cyathiformis (Bosc) Morgan (BTC, T, C, M)

Lycoperdon umbrinum Pers. (BPE, T, C, M)

\section{SCLERODERMATALES}

Astraeaceae

Astraeus hygrometricus (Pers.) Morgan (BPE, T, M)

Sclerodermataceae

Pisolithus arhizus (Scop, : Pers.) Rauschert (BPE, T, M, V)

Scleroderma areolatum Ehrenb. (BMM, T, M, V)

S. texense Berk. (BP, BPE, BMM, T, M, V)

S. verrucosum (Bull. : Pers.) Pers. (BPE, T, M, V)

Veligaster nitidus (Berk.) Guzmán \& Tapia (BE, BMM, T, M, V)

PHALLALES

Phallaceae

Phallus hadriani Vent. : Pers. (BEP, BMM, T, M)

\section{AURICULARIALES}

Auriculariaceae

Auricularia auricula-judae (Bull. : Fr.) Wettst. (BMM, VS, L, C, DM)

A. delicata (Fr.) J. F. Hennen (BP, BMM, L, C, DM)

A. mesenterica Pers. (BMM, L, DM)

A. polytricha (Mont.) Sacc. (BP, BMM, BTC, L, C, DM)

\section{DACRYMYCETALES}

Dacrymycetaceae

* Calocera cornea (Bastch : Fr.) Fr. (BPE, BMM, L, DM) Dacrymyces palmatus (Schwein.) Bres. (BPE, L, DM)

Dacryopinax spathularia (Schwein.) G. W. Martin (BPE, BTC, BTS, L, DM)

* D. elegans (Berk. \& Curtis) G. W. Martin (BTS, L, DM)

\section{TREMELLALES}

Tremellaceae

Tremella concrescens (Fr.) Burt. (BMM, L, DM)

* $T$. aff. dumontii Lowy (BMM, L, DM)

T. fuciformis Berk. (BEP, L, DM)

* T. mesenterica (Retz.) Fr. (BPE, L, DM) 
Apéndice. Continuación.

\section{TELIOMYCETES \\ UREDINALES \\ Cronartium conigenum Hedg. \& N. R. Hunt (BP, Fi, P)}

Simbología utilizada:

* Nuevo registro para el estado de Jalisco, ** Nuevo registro para México, BP bosque de pino, BE bosque de encino, BEP bosque de encino-pino, BPE bosque de pino-encino, BMM bosque mesófilo de montaña, BTC bosque tropical caducifolio, BTS bosque tropical subcaducifolio, VS vegetación secundaria, F fimícola, Fi fitopatógeno, Fu fungícola, H humícola, I entomopatógeno, L lignícola, T terrícola, A alucinógeno, C comestible, DM destructor de madera, $\mathrm{M}$ micorrízico, $\mathrm{P}$ parásito, $\mathrm{V}$ venenoso.

\section{AGRADECIMIENTOS}

Las autoras expresan su agradecimiento a CONABIO a través de los proyectos G013 y 011, a SIMORELOS - CONACYT, proyecto 96-06-002, a Idea Wild y a la Universidad de Guadalajara, por el financiamiento otorgado que contribuyó a la realización de este trabajo. Se agradece al Dr. Gastón Guzmán, del Instituto de Ecología de Xalapa, Ver., por sus sugerencias y la revisión del manuscrito y al Dr. Teuvo Ahti de la Universidad de Helsinki, por la revisión nomenclatural del listado de las especies. Al M. en C. Ricardo Valenzuela se le reconoce ampliamente por su colaboración para la identificación de algunos de los Poriales aquí incluidos. Asimismo se agradece a Manuel Flores por la elaboración de los dibujos de los cuerpos fructíferos y a Miguel De Santiago por entintar los dibujos de las estructuras microscópicas. A la Biól. Teresa Cuevas Arias se le da las gracias por la elaboración del mapa de la zona.

\section{LITERATURA CITADA}

Anónimo. 1988. Los municipios de Jalisco. Colección: Enciclopedia de los municipios de México. Centro Estatal de Estudios Municipales de Jalisco. Guadalajara. 837 pp.

Bandala-Muñoz, V. M., G. Guzmán y L. Montoya-Bello. 1988. Especies de macromicetos citadas de México, VII. Agaricales, Parte II (1972-1987). Rev. Mex. Mic. 4: 205-250.

Bandala-Muñoz, V. M., G. Guzmán y L. Montoya. 1993. Los hongos del grupo de los Poliporáceos conocidos en México. Reporte Científico, Facultad de Ciencias Forestales, Universidad Autónoma de Nuevo León, No. especial 13: 1-55.

Bandala-Muñoz, V. M., L. Montoya-Bello y G. Guzmán. 1987a. Especies de macromicetos citadas de México, VI. Tremellales y Aphyllophorales (excluyendo Polyporaceae), parte II. Rev. Mex. Mic. 3: $161-174$. 
Bandala-Muñoz, V. M., L. Montoya-Bello y G. Guzmán. 1987b. Nuevos registros de hongos del estado de Veracruz, III. Descripción de algunos Ascomycetes y Aphyllophorales (con nuevos registros para los estados de Hidalgo, Morelos y Tlaxcala). Rev. Mex. Mic. 3: 51-69.

Bautista, N., S. Chacón y G. Guzmán. 1986. Ascomycetes poco conocidos en México, III. Especies del estado de Morelos. Rev. Mex. Mic. 2: 85-104.

Breitenbach, J. y F. Kränzlin. 1986. Fungi of Switzerland, Vol. 2. Verlag Mykologia, Lucerna. 411 pp.

Cifuentes, J., M. Villegas, L. Pérez-Ramírez, M. Bulnes, V. Corona, M. del R. González, I. Jiménez, A. Pompa y G. Vargas. 1990. Observaciones sobre la distribución, hábitat e importancia de los hongos de Los Azufres, Michoacán. Rev. Mex. Mic. 6: 11-28.

Coker, C. N. 1974. The club and coral mushrooms (Clavarias) of the United States and Canada. Dover. Nueva York. 209 pp.

Chacón, S. y R. Medel. 1993. Los hongos (principalmente macromicetos) registrados en el bosque mesófilo de montaña de México. Reporte Científico, Facultad de Ciencias Forestales, Universidad Autónoma de Nuevo León. No. especial 13: 61-110.

Dennis, R. W. G. 1970. Fungus flora of Venezuela and adjacent countries. Cramer. Lehre. 531 pp.

Díaz-Barriga, H., F. Guevara-Fefer y R. Valenzuela. 1988. Contribución al conocimiento de los macromicetos del estado de Michoacán. Acta Bot. Mex. 2: 21-24.

Fierros, M. L. y L. Guzmán-Dávalos. 1995. Inventario preliminar de los hongos macroscópicos de la Sierra de Quila, Jalisco, México. Bol. IBUG (Instituto de Botánica, Universidad de Guadalajara) 3(1-3): 129-142.

Frutis, I. y G. Guzmán. 1983. Contribución al conocimiento de los hongos del estado de Hidalgo. Bol. Soc. Mex. Mic. 18: 219-265.

Gilbertson, R. L. y L. Ryvarden. 1986. North American Polypores, 1. Abortiporus-Lindtneria. Fungiflora, Oslo. pp. 1-433.

Gilbertson, R. L. y L. Ryvarden. 1987. North American Polypores, 2. Megasporoporia-Wrightoporia. Fungiflora, Oslo. pp. 437-885.

Guzmán, G. 1977. Identificación de los hongos. Comestibles, venenosos, alucinantes y destructores de la madera. Limusa. México, D. F. 452 pp.

Guzmán, G. y D. A. García-Saucedo. 1973. Macromicetos del estado de Jalisco I. Consideraciones generales y distribución de las especies conocidas. Bol. Soc. Mex. Mic. 7: 129-143.

Guzmán, G. 1998. Las especies de Psilocybe (Fungi, Basidiomycotina, Agaricales) conocidas de Jalisco (México) y descripción de dos nuevas para la ciencia. Acta Bot. Mex. 43: 23-32.

Guzmán-Dávalos, L. y G. Guzmán. 1979. Estudio ecológico comparativo entre los hongos (macromicetos) de los bosques tropicales y los de coníferas del sureste de México. Bol. Soc. Mex. Mic. 13: 89-126.

Guzmán-Dávalos, L. y G. Guzmán. 1985. Hongos del Estado de Jalisco V. El género Scleroderma. Rev. Mex. Mic. 1: 109-128.

Guzmán-Dávalos, L. y G. Guzmán. 1986. Hongos de Jalisco. VII. El género Gymnopilus (Cortinariaceae). Rev. Mex. Mic. 2: 157-185.

Guzmán-Dávalos, L. y G. Guzmán. 1991. Additions to the genus Gymnopilus (Agaricales, Cortinariaceae) from Mexico. Mycotaxon 41: 43-56.

Hawksworth, D. L., P. M. Kirk, B. C. Sutton y D. N. Pegler. 1995. Ainsworth \& Bisby's dictionary of the fungi. $8^{\circ}$ ed. $C A B$ International. Cambridge. $616 \mathrm{pp}$.

Largent, D., D. Johnson y R. Watling, 1977. How to identify mushrooms to genus III: microscopic features. Mad River Press. Eureka. 148 pp.

Lowy, B. 1971. Tremellales. Flora Neotropica 6. Hafner. Nueva York. 153 pp.

Lowy, B. 1980. Tremellales. Flora Neotropica 6 (supplement). New York Botanical Garden. Nueva York. $18 \mathrm{pp}$.

Manzi, J. 1976. Hongos. Contribución al conocimiento de las especies comestibles y venenosas del área central del estado de Jalisco, México. Ed. Combonianas. Guadalajara. 119 pp. 
Herrera et al.: Contribución al Conocimiento de la Micobiota de San Sebastián del Oeste, Jalisco

Marmolejo, J. G., J. Castillo y G. Guzmán. 1981. Descripción de Telephoraceos poco conocidos en México. Bol. Soc. Mex. Mic. 15: 9-66.

Martin, G. N. 1969. Revision of the north central Tremellales. Cramer. Lehre. $122 \mathrm{pp.}$

Medel, R., G. Guzmán y S. Chacón. 1999. Especies de macromicetos citadas de México IX. Ascomycetes, parte III: 1983-1996. Acta Bot. Mex. 46: 57-72.

Murrill, W. A. 1973. Tropical polypores. Cramer. Lehre. 113 pp.

Nieves, G. 1985. Contribución al conocimiento de los macromicetos del Bosque La Primavera, Zapopan, Jal. Tesis profesional. Facultad de Agronomía, Universidad de Guadalajara. Guadalajara, Jal. $74 \mathrm{pp}$.

Ojeda-López, S., M. L. Sandoval y R. Valenzuela. 1986. Los poliporáceos de México I. Descripción de algunas especies del noreste de Guanajuato. Rev. Mex. Mic. 2: 367-436.

Pérez-Ramírez, L., M. Villegas y J. Cifuentes. 1986. Descripción de macromicetos poco estudiados en México II. Rev. Mex. Mic. 2: 251-257.

Petersen, H. R. 1988. The clavariod fungi of New Zealand. Wellington. Nueva Zelanda. 170 pp.

Phillips, R. 1981. Mushrooms and other fungi of Great Britain \& Europe. Pan Books. Londres. $287 \mathrm{pp}$.

Phillips, R. 1991. Mushrooms of North America. Little, Brown Co. Boston. 319 pp.

Reynoso, J. J. y J. Curiel. 1977. San Sebastián del Oeste, Jalisco, In: Curiel, B. A. (comp.) Áreas naturales prioritarias para la conservación en la región II. Secretaría del Medio Ambiente, Recursos Naturales y Pesca. México, D.F. pp. 21-22.

Rodríguez, O. 1998. Avances en el conocimiento del género Pluteus (Fungi, Agaricales) en Nueva Galicia, México. Bol. IBUG (Instituto de Botánica, Universidad de Guadalajara) 5(1-3): 87-93.

Rodríguez, O. y L. Guzmán-Dávalos. 1993. Nuevos registros de hongos para Jalisco. Bol. IBUG (Instituto de Botánica, Universidad de Guadalajara) 1(5): 333-338.

Rodríguez, O. y L. Guzmán-Dávalos. 1997. New additions to the genus Pluteus (Pluteaceae, Agaricales) for Mexico. Micol. Neotrop. Apl. 10: 83-91.

Rodríguez, O., M. Garza y L. Guzmán-Dávalos. 1994. Inventario preliminar de los hongos del Volcán de Tequila, estado de Jalisco. Rev. Mex. Mic. 10: 103-111.

Rodríguez, O., O. Vargas y L. Guzmán-Dávalos. 1997. New reports of the genus Pluteus (Agaricales) from Mexico. Mycotaxon 61: 473-480.

San Martín, F., Y. M. Ju y J. D. Rogers. 1999. Algunas especies de Hypoxylon (Pyrenomycetes, Xylariaceae) de México. Acta Bot. Mex. 47: 31-53.

Sánchez-Jácome, M. y L. Guzmán-Dávalos. 1997. Nuevos registros de Thelephora (Aphyllophorales, Basidiomycotina) para México. Rev. Mex. Mic. 13: 70-77.

Sierra, S. y J. Cifuentes. 1993. Contribución al estudio taxonómico de los hongos tremeloides (Heterobasidiomycetes) de México. Rev. Mex. Mic. 9: 119-137.

Singer, R. 1986. The Agaricales in modern taxonomy. 4ª ed. Koeltz. Koenigstein. 981 pp. + 88 láms.

Singer R., J. García y L. D. Gómez. 1992. The Boletineae of Mexico and Central America IV. Beih. Nova Hedwigia 105: 1-62.

Téllez, C., L. Guzmán-Dávalos y G. Guzmán. 1988. Contribución al conocimiento de los hongos de la Reserva de la Biosfera de la Sierra de Manantlán, Jalisco. Rev. Mex. Mic. 4: 123-130.

Vázquez, L. S. y L. Guzmán-Dávalos. 1988. Algunas especies de hongos de la Barranca de Huentitán, estado de Jalisco. Rev. Mex. Mic. 4: 75-88.

Vázquez, L. S. y L. Guzmán-Dávalos. 1991a. Nuevos registros de polipóraceos estipitados de Jalisco. Rev. Mex. Mic. 7: 113-120.

Vázquez, L. S. y L. Guzmán-Dávalos. 1991b. Nuevos registros de basidiomycetes para Jalisco. Bol. IBUG (Instituto de Botánica, Universidad de Guadalajara) 4(3-1): 123-130.

Vázquez, L. S. y L. Guzmán-Dávalos. 1991c. Los hongos del género Volvariella (Agaricales, Basidiomycetes) conocidos en Jalisco. Bol. IBUG (Instituto de Botánica, Universidad de Guadalajara) 1(1): 15-22. 
Vázquez, L. S., L. Guzmán-Dávalos y G. Guzmán. 1989. Contribución al conocimiento de las especies del género Volvariella en Jalisco. Rev. Mex. Mic. 5: 169-179.

Villarruel-Ordaz, J. L., L. Pérez-Ramírez y J. Cifuentes. 1993. Nuevos registros del género Collybia (Tricholomataceae) y descripción de especies poco estudiadas en México. Rev. Mex. Mic. 9: 139-164.

Welden, A. L. y G. Guzmán. 1978. Lista preliminar de los hongos, líquenes y macromicetos de las regiones de Uxpanapa, Coatzacoalcos, Los Tuxtlas, Papaloapan y Xalapa (parte de los Estados de Veracruz y Oaxaca). Bol. Soc. Mex. Mic. 12: 59-102.

Welden, A. L., L. Dávalos y G. Guzmán. 1979. Segunda lista de los hongos, líquenes y mixomicetos de las regiones de Uxpanapa, Coatzacoalcos, Los Tuxtlas, Papaloapan y Xalapa (México). Bol. Soc. Mex. Mic. 13: 151-161. 